\title{
EDITORIAL
}

\section{Theoretical ecology: a successful first year and a bright future for a new journal}

\author{
Alan Hastings \\ Published online: 17 February 2009 \\ C The Author(s) 2009. This article is published with open access at Springerlink.com
}

This issue marks the start of volume 2 of Theoretical Ecology. Looking back, this has been an extraordinarily successful first year. Looking forward, it is clear that a journal focusing on theoretical ecology can play an expanding role in ecology. Questions about the need for a journal in this area have been answered by the overall interest in the journal. Any questions about the desirability of a journal in theoretical ecology have been answered by the continuing flow of submissions of high quality manuscripts across a breadth of topics.

One way to sum up the success of this first year is through numbers. All four issues of the journal appeared as scheduled, with a total of 22 papers. A very positive feature of these initial contributions is that almost all span several areas of ecology. However, to give a rough feel for the breadth of the publications in the journal, I have tried to put each paper into a single category, leading to the following very crude classification. Five papers were in the area of ecosystem or population management, 4 in community ecology, 4 in population dynamics, 4 in philosophical or methodological areas (including statistics and model analysis), 3 in evolutionary ecology, and 2 in the area of networks or foodwebs. This variety and balance of contributions is very encouraging. Two papers were featured in the Faculty of 1000 list of noteworthy papers.

Other numbers tell an encouraging story as well. At this time, we have had 99 submissions, with a final decision on 75. Thirty-seven manuscripts have been accepted, so the

A. Hastings $(\square)$

Department of Environmental Science and Policy,

University of California,

Davis, CA 95616, USA

e-mail: amhastings@ucdavis.edu acceptance rate is just under $50 \%$. The journal has also been rapid in reaching decisions, with the average time to first decision for initial submissions of 52 days. For first revisions, the average time to decision was 28 days. This achievement has only been possible because of the very generous efforts by reviewers and editorial board members.

Ecology is an international science and a goal of the journal is to reflect this. The papers published in volume 1 do display a geographic breadth. Based just on the correspondence address of the first author, only slightly over half the papers (13) are from US institutions. There are 3 papers from Canada, 2 from the UK, 2 from other parts of Europe, and 1 each from Japan and South America.

The journal will continue to encourage submissions across the breadth of theoretical ecology. Contributions that are truly interdisciplinary in nature will be particularly welcome. Notably, papers published this first past year included a significant number focusing on applications. This promising development can be traced back to some of the earliest work in theoretical ecology such as Volterra's attention to predator-prey cycles based on oscillations in fish species, or to initial investigations into epidemics. Given increasing need for and attention paid to management of natural systems, theory focusing on applications and management is becoming an even more central part of ecology. The journal Theoretical Ecology will play a key role in these developments.

Looking forward, there will be exciting changes in the journal in the coming year. Papers identified as Brief Communications that will be reviewed even more quickly will appear. This year will also see the publication of review papers here for the first time. Theoretical ecology as a discipline is one where reviews can be particularly valuable, as it is important to focus not only on specific systems and models, but on conclusions that range across 
systems and different formulations. Use the reviews that will appear during the coming year as a guide to the kinds of manuscripts we will look for, but do not be limited by the topics or format. Contact me if you have questions about appropriate topics for review articles.

Finally, I would like to express my thanks to many of the people who have made this first year so successful. As I mentioned above, the willingness of reviewers to provide the expertise needed has been impressive. The editorial board has been supportive. The support of Tamara Welschot and the team at Springer has also been superb. Jill Poloske at Davis has managed superbly communication with authors and reviewers. The response of readers has been encouraging. I am grateful to all.

Open Access This article is distributed under the terms of the Creative Commons Attribution Noncommercial License which permits any noncommercial use, distribution, and reproduction in any medium, provided the original author(s) and source are credited. 